\title{
Impact of timing of initiation of dialysis on mortality of patients with acute kidney injury
}

\author{
Reginaldo Passoni dos Santos ${ }^{1,2^{*}}{ }^{\oplus}$, Letícia Giroldo Vieira ${ }^{3}{ }^{\oplus}$, Danielle Fernanda Miner de Oliveira ${ }^{3}$, Raissa Fritz \\ Schmitt $^{3^{\oplus}}$, Vinicius Ferreira de Barros ${ }^{3 \oplus}$, Ariana Rodrigues da Silva Carvalho ${ }^{2,4}$, Luis Alberto Batista Peres ${ }^{2,5}$
}

\begin{abstract}
Introduction: In Brazil, primary studies on this issue are still limited and the ideal timing of initiation of dialysis in severe acute kidney injury (AKI) still generates disagreements among experts.

Objectives: To assess if the timing of initiation of dialysis is associated with the mortality of patients with AKI in intensive care unit (ICU). Patients and Methods: We retrospectively analyzed medical records of patients that developed severe AKI in the ICU. Bivariate analysis was carried out to compare data between groups of patients who underwent early dialysis (ED - initiated up to two days after the AKI diagnosis) and late dialysis (LD - initiated more than two days after the AKI diagnosis), while multivariate logistic regression was applied to identify factors associated with mortality.

Results: Of the 76 patients included in the study, 27 (35.5\%) were allocated in the ED group and $49(64.5 \%)$ in the LD group. LD group had a higher frequency of sepsis [26 (53\%) vs. $12(44 \%) ; P=0.472$, while the ED group had a higher median number of dialysis sessions ( 6 vs. $3 ; P=0.477)$ and higher total median time on dialysis $(17.5 \mathrm{~h}$ vs. $13 \mathrm{~h} ; P=0.629)$. The overall mortality rate was $61.8 \%(\mathrm{n}=47)$ and of $76 \%(n=22)$ in the ED group. The patients' serum creatinine level at admission in the ICU was the only statistically significant risk factor for death $[\mathrm{OR}=0.453(95 \% \mathrm{Cl}=0.257-0.801) ; P=0.006]$.

Conclusion: The overall and in the ED group mortality rate was elevated, however, the timing of initiation of dialysis did not show statistically significant association with death. The serum creatinine at ICU admission seems to be an important mortality predictor. Keywords: Acute kidney injury, Renal replacement therapy, Mortality, Dialysis, Risk factors, Intensive care unit

Citation: Passoni dos Santos R, Giroldo Vieira L, Fernanda Miner de Oliveira D, Fritz Schmitt R, Ferreira de Barros V, Rodrigues da Silva Carvalho A, Alberto Batista Peres L. Impact of timing of initiation of dialysis on mortality of patients with acute kidney injury. J Renal Endocrinol. 2021;7:e07. doi: 10.34172/jre.2021.07

Copyright ( $\odot 2021$ The Author(s); Published by Nickan Research Institute. This is an open-access article distributed under the terms of the Creative Commons Attribution License (http://creativecommons.org/licenses/by/4.0), which permits unrestricted use, distribution, and reproduction in any medium, provided the original work is properly cited.
\end{abstract}

\section{Introduction}

The dialysis therapy is one of the main management strategies for patients with acute kidney injury (AKI) in intensive care unit (ICU) (1-5). Studies suggest that early initiation of dialysis in these patients reduces adverse renal events and prevents metabolic complications $(1,2)$. On other hand, late initiation appears to be an option with some benefit when assessing the possibility of recovery of renal function after AKI without the need for complex, potentially dangerous, costly treatment with limited availability in developing countries $(3,4)$.

Given the impact on patients' prognosis and clinical outcomes, the decision on when to start dialysis in patients with severe AKI is crucial $(1,5)$. In 2016, Gaudry et al (6), at France, and Zarbock et al (7), at Germany published data from two studies on the impact of timing of early dialysis (ED) initiation on the mortality of critical ill AKI patients. In this same sense, Brazilian researchers conducting a systematic review, concluding that the early initiation of dialysis does not attribute greater survival to patients with severe AKI (8).

\section{Objectives}

Notwithstanding, despite the valuable contributions added to the literature, in Brazil, primary studies on this issue are still limited and the ideal timing of initiation of dialysis in severe AKI still generates disagreements among experts. Like this, remains without an established consensus. Therefore, this study aimed to asses if the timing of initiation of dialysis is associated with the mortality of patients with AKI in ICU.

Patients and Methods

Study design

The study protocol was previously approved by the research ethics committee of the Western State University

${ }^{1}$ Department of Nursing, Teaching Hospital, Western Paraná State University, Cascavel, PR, Brazil. ${ }^{2}$ Postgraduate Program in Biosciences and Health, Western Paraná State University, Cascavel, PR, Brazil. ${ }^{3}$ Undergraduate Course in Medicine, FAG University Center, Cascavel, PR, Brazil. ${ }^{4}$ Collegiate of Nursing, Western Paraná State University, Cascavel, PR, Brazil. ${ }^{5}$ Discipline of Nephrology, Undergraduate Course in Medicine, Western Paraná State University, Cascavel, PR, Brazil.

*Corresponding Author: Reginaldo Passoni dos Santos, Email: regi-pas@hotmail.com 
Implication for health policy/practice/research/ medical education

The timing of initiation of dialysis is one of the main dilemmas of clinical practice and, in this study, did not show statistically significant association with death. On the other hand, the serum creatinine level, presented by patients at the time of ICU admission, was shown to be a risk factor independently associated with death. However, more Brazilian studies need to be conducted and with a more appropriate methodological design, in order to verify the real impact of the moment of dialysis on the mortality of patients in our country.

Parana. The recommendations of STrengthening the Reporting of OBservational studies in Epidemiology (STROBE) (9) guidelines were flowed.

\section{Design, setting and participants}

We carried out a retrospective study, through the review of medical records of patients with severe AKI, hospitalized between January 2011 and December 2016, in the mixed ICU of a university hospital located in the Brazil's Southern region. Our ICU consists of 14 beds to hospitalization of critically ill patients with clinical and surgical conditions. For inclusion on study, were selected medical records of patients with 18 years or older, with a minimum stay in the unit of 48 hours and, who undergoing dialysis due to occurrence of severe AKI in the ICU, carried out by kidney disease improving global outcomes (KDIGO) criteria (10). The records were separated in two groups, according to the patients' timing of initiation of dialysis. Patients with initiation of dialysis within two days after AKI diagnosis were allocated in the ED group, while patients with initiation of dialysis more than two days after the AKI diagnosis were allocated in the late dialysis (LD) group.

\section{Variables and outcome}

The data were retrieved in February 2018, using a semistructured form prepared by the authors. Information were collected regarding to socio-demographic (gender; age; race; comorbidities), admission data (previous unit; diagnosis clinical or surgical; elective or emergency admission; serum creatinine; urine output in the first 24 hours and mechanical ventilation at admission); acute physiology and chronic health evaluation II (APACHE II) (11) and sequential organ failure assessment (SOFA) (12) scores, as well as length of ICU stay also were retrieved.

In addition, we collected information about events that occurred in the ICU (days on mechanical ventilation; infection and sepsis occurrence; blood transfusions and need for surgical procedures) and data from dialysis (number of sessions; total time of dialysis in hours; intradialytic intercurrences and need of interruption).

Mortality rate was the outcome of interest and, the risk factors for mortality were identified.

\section{Ethical issues}

The research followed the tenets of the Declaration of Helsinki. The Ethics Committee of Western Paraná State University approved this study. The institutional ethical committee at Western Paraná State University approved all study protocols (Approval number\#1.622.962). Accordingly, written informed consent was taken from all participants before any intervention. This study was extracted from MSc thesis of Postgraduate Program in Biosciences and Health at this university (Thesis "Acute kidney injury in intensive care unit; a longitudinal study").

\section{Statistical analysis}

The data were tabulated in a Microsoft Excel ${ }^{\circ}$ spreadsheet and analyzed using XLSTAT software, version 2018. After analyzing the assumptions normality (Shapiro-Wilk test) and homoscedasticity ( $F$ test), continuous variables were expressed by the mean and standard derivation or median and inter-quartile range, while categorical variables were presented by the gross (n) and relative (\%) frequencies.

The crude mortality rate was calculated and according to timing of initiation of dialysis. We compared the data between ED and LD groups, as well as between survivors and non-survivors applying the chi-square and/or Fisher's exact tests to the categorical variables and the student's $t$ test or Mann-Whitney U test to the continuous variables, as appropriate.

Variables that in the bivariate analysis had a $P$ value $<0.20$ were included in the multivariate logistic regression model, to analyze the risk factors independently associated with death and the variables in the final multivariate model were selected using the stepwise method. To avoid selection bias, variables with an inflation factor of variance greater the five were excluded from the final model, which had its performance evaluated by the Hosmer-Lemeshow test and the area under the receiver operation characteristic curve. In all tests, $P$ value $<0.05$ was considered statistically significant.

\section{Results}

During the study period, 613 patients were diagnosed with AKI in the ICU, of whom $238(38.8 \%)$ had severe AKI and, of these, the 76 (31.9\%) undergoing dialysis were included in the present study. All patients (100\%) undergoing intermittent hemodialysis and were allocated to the $\operatorname{ED}(\mathrm{n}=27 ; 35.5 \%)$ and $\mathrm{LD}(\mathrm{n}=49 ; 64.5 \%)$. The median age of the patients was $52(43-62)$ years and the majority were male $(n=42 ; 55 \%)$ and Caucasian $(n=66$; $87 \%)$.

Urine output in the first 24 hours of admission to the ICU was lower in the ED group $(350 \mathrm{~mL}$ vs. $1600 \mathrm{~mL}$; $P \leq$ 0.001 ), while the median of SOFA score was lower in the LD group (11 points vs. 13 points; $P=0.019$ ). LD group needed more time on mechanical ventilation (15 days vs. 
13 days; $P=0.377)$, and a higher frequency of sepsis [26 (53\%) vs. 12 (44\%); $P=0.472$ ]. Regarding the dialysis data, it was verified that the ED group had a higher median number of dialysis sessions ( 6 vs. $3 ; P=0.477$ ) and higher total median time on dialysis ( $17.5 \mathrm{~h}$ vs. $13 \mathrm{~h} ; P=0.629)$. The need to interrupt the dialysis session was similar in both groups [24 (49\%) to ED group vs. 13 (48\%) to LD group; $P=0.996$ ) (Table 1).

The overall mortality rate was $61.8 \%(n=47)$ and of $76 \%(n=22)$ in the ED group. In the bivariate analysis, survivors patients had higher serum creatinine levels (2.16 mg/dL vs. $1.68 \mathrm{mg} / \mathrm{dL} ; P=0.038)$, higher frequency of infection [24 (83\%) vs. 25 (53\%); $P=0.009$ ], longer ICU stay (28 days vs. 17 days; $P=0.006)$ and, required more dialysis sessions ( 7 vs. 3 ; $P=0.034$ ) (Table 2 ). By multivariate logistic regression, we verified that the patients' serum creatinine level at admission in the ICU was the only statistically significant risk factor for death in the studied sample (Table 3).

\section{Discussion}

This retrospective and single-center study presented the general features of a sample of patients with severe AKI in the ICU and evaluated the impact of timing of initiation of dialysis on the mortality of patients. With regards to the clinical-epidemiological profile of the patients, there was some homogeneity between the ED and LD groups, with no statistically significant differences for most variables (Table 1). Still this, we found that patients in the LD group had lower serum creatinine levels at ICU admission and greater urine output in the first 24 hours. Considering that such variables make up the KDIGO (10) criteria, possibly, besides $\mathrm{LD}$, characterized by initiation two days after AKI diagnosis, we believe, empirically, that the AKI diagnosis itself, in patients of LD group, also occurred later, compared to the patients in the ED group.

Table 1. Features of patients, according with the timing of initiation of dialysis

\begin{tabular}{|c|c|c|c|c|}
\hline Variables & All $(n=76)$ & ED $(n=27)$ & LD $(n=49)$ & $P$ \\
\hline Gender (male) & $42(55 \%)$ & $15(56 \%)$ & $27(55 \%)$ & $0.970^{\mathrm{a}}$ \\
\hline Age & $52(43-62)$ & $54(40-63)$ & $50(43-60)$ & $0.769^{b}$ \\
\hline Race (Caucasian) & $66(87 \%)$ & $24(89 \%)$ & $42(86 \%)$ & $0.695^{a}$ \\
\hline Hypertension & $28(37 \%)$ & $11(41 \%)$ & $17(35 \%)$ & $0.601^{\mathrm{a}}$ \\
\hline Diabetes mellitus & $18(24 \%)$ & $3(11 \%)$ & $15(31 \%)$ & $0.056^{\mathrm{a}}$ \\
\hline Source unit & & & & $0.624^{c}$ \\
\hline Emergency room & $51(67 \%)$ & $20(74 \%)$ & $31(64 \%)$ & \\
\hline Surgical center & $16(21 \%)$ & $4(15 \%)$ & $12(24 \%)$ & \\
\hline Ward & $9(12 \%)$ & $3(11 \%)$ & $6(12 \%)$ & \\
\hline Admission diagnosis & & & & $0.374^{\mathrm{a}}$ \\
\hline Clinical & $37(49 \%)$ & $15(56 \%)$ & $22(45 \%)$ & \\
\hline Surgical & $39(51 \%)$ & $12(44 \%)$ & $27(55 \%)$ & \\
\hline Admission type & & & & $0.434^{a}$ \\
\hline Elective & $21(28 \%)$ & $6(22 \%)$ & $15(31 \%)$ & \\
\hline Urgency & $55(72 \%)$ & $21(78 \%)$ & $34(69 \%)$ & \\
\hline Serum creatinine $(\mathrm{mg} / \mathrm{dL})$ at ICU admission & $1.9(1.1-2.5)$ & $2.1(1.4-3.2)$ & $1.8(1.1-2.3)$ & $0.068^{\mathrm{b}}$ \\
\hline Urine output at first 24 hours & $1212(326-2225)$ & $350(137-1190)$ & $1600(680-2400)$ & $<0.001^{\text {b }}$ \\
\hline Mechanical ventilation need & $68(89 \%)$ & $24(89 \%)$ & $44(90 \%)$ & $0.902^{\mathrm{a}}$ \\
\hline Mechanical ventilation time (days) & $14(8-26)$ & $13(6-29,5)$ & $15(9-26)$ & $0.397^{b}$ \\
\hline Infection & $49(64 \%)$ & $14(52 \%)$ & $35(71 \%)$ & $0.088^{\mathrm{a}}$ \\
\hline Sepsis & $38(50 \%)$ & $12(44 \%)$ & $26(53 \%)$ & $0.472^{\mathrm{a}}$ \\
\hline Blood transfusion & $56(74 \%)$ & $17(63 \%)$ & $39(80 \%)$ & $0.115^{a}$ \\
\hline Surgery & $50(66 \%)$ & $15(56 \%)$ & $35(71 \%)$ & $0.163^{\mathrm{a}}$ \\
\hline \multicolumn{5}{|l|}{ Data from dialysis } \\
\hline Number of sessions & $4(2-10)$ & $6(2-10)$ & $3(2-9)$ & $0.487^{b}$ \\
\hline Total time $(h)$ & $13.5(6-29.3)$ & $17.5(6.5-30.5)$ & $13(6-28)$ & $0.629^{b}$ \\
\hline Intradialytic complications & $3(2-4)$ & $2(1-4)$ & $3(2-4)$ & $0.945^{b}$ \\
\hline Need of interruption & $37(49 \%)$ & $13(48 \%)$ & $24(49 \%)$ & $0.996^{\mathrm{a}}$ \\
\hline APACHE II & $31(26-34)$ & $32(29-35)$ & $29(25-33)$ & $0.075^{\mathrm{b}}$ \\
\hline SOFA & $12(10-14)$ & $13(10-15)$ & $11(9-13)$ & $0.019^{b}$ \\
\hline Length ICU stay & $21(11-36)$ & $18(6.5-36)$ & $25(14-36)$ & $0.193^{b}$ \\
\hline
\end{tabular}

ED: Early Dialysis; LD: Late dialysis; APACHE II: Acute Physiology and Chronic Health Evaluation II; SOFA: Sequential Organ Failure Assessment; ICU: Intensive Care Unit;

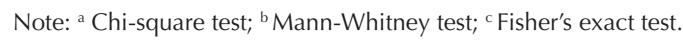


Table 2. Features of patients, according with the mortality

\begin{tabular}{|c|c|c|c|}
\hline Variables & Survivor $(n=29)$ & Non-survivor $(n=47)$ & $P$ \\
\hline Gender (male) & $14(48 \%)$ & $28(60 \%)$ & $0.336^{a}$ \\
\hline Age & $48(43-62)$ & $54(42-61)$ & $0.653^{b}$ \\
\hline Race (Caucasian) & $26(90 \%)$ & $40(85 \%)$ & $0.569^{\mathrm{a}}$ \\
\hline Hypertension & $12(41 \%)$ & $16(34 \%)$ & $0.520^{\mathrm{a}}$ \\
\hline Diabetes mellitus & $11(38 \%)$ & $7(15 \%)$ & $0.022^{\mathrm{a}}$ \\
\hline Source unit & & & $1.000^{c}$ \\
\hline Emergency room & $20(69 \%)$ & $31(66 \%)$ & \\
\hline Surgical center & $6(21 \%)$ & $10(21 \%)$ & \\
\hline Ward & $3(10 \%)$ & $6(13 \%)$ & \\
\hline Admission diagnosis & & & $0.677^{a}$ \\
\hline Clinical & $15(52 \%)$ & $22(47 \%)$ & \\
\hline Surgical & $14(48 \%)$ & $25(53 \%)$ & \\
\hline Admission type & & & $0.288^{\mathrm{a}}$ \\
\hline Elective & $6(21 \%)$ & $15(32 \%)$ & \\
\hline Urgency & $23(79 \%)$ & $32(68)$ & \\
\hline Serum creatinine $(\mathrm{mg} / \mathrm{dL})$ at ICU admission & $2.16(1.45-3.39)$ & $1.68(0.99-2.29)$ & $0.038^{\mathrm{b}}$ \\
\hline Urine output at first 24 hours & $1.275(300-1.755)$ & $1.150(337-2.300)$ & $0.646^{\mathrm{b}}$ \\
\hline Mechanical ventilation need & $26(90 \%)$ & $42(89)$ & $0.968^{\mathrm{a}}$ \\
\hline Mechanical ventilation time (days) & $17(11-26)$ & $13(7-27)$ & $0.248^{\mathrm{b}}$ \\
\hline Infection & $24(83 \%)$ & $25(53 \%)$ & $0.009^{\mathrm{a}}$ \\
\hline Sepsis & $13(45 \%)$ & $25(53 \%)$ & $0.479^{\mathrm{a}}$ \\
\hline Blood transfusion & $21(72 \%)$ & $35(74 \%)$ & $0.843^{\mathrm{a}}$ \\
\hline Surgery & $21(72 \%)$ & $29(62 \%)$ & $0.339^{\mathrm{a}}$ \\
\hline \multicolumn{4}{|l|}{ Data from dialysis } \\
\hline Number of sessions & $7(2-11)$ & $3(1-7)$ & $0.034^{b}$ \\
\hline Total time (hours) & $21(7-36)$ & $12(4-24)$ & $0.100^{\mathrm{b}}$ \\
\hline Intradialytic complications & $8(28 \%)$ & $29(62 \%)$ & $0.339^{\mathrm{a}}$ \\
\hline Need of interruption & $11(38 \%)$ & $26(55 \%)$ & $0.141^{\mathrm{a}}$ \\
\hline Timing of initiation of dialysis & & & $0.103^{\mathrm{a}}$ \\
\hline ED & $7(23 \%)$ & $22(76 \%)$ & \\
\hline LD & $20(43 \%)$ & $27(57 \%)$ & \\
\hline APACHE II & $32(27-36)$ & $30(25-33)$ & $0.167^{\mathrm{b}}$ \\
\hline SOFA & $12(10-14)$ & $11(10-13)$ & $0.471^{\mathrm{b}}$ \\
\hline Length ICU stay & $28(19-37)$ & $17(8-28)$ & $0.006^{b}$ \\
\hline
\end{tabular}

ED: Early Dialysis; LD: Late dialysis; APACHE II: Acute Physiology and Chronic Health Evaluation II; SOFA: Sequential Organ Failure Assessment; ICU: Intensive Care Unit;

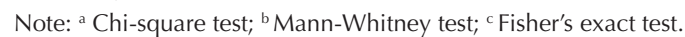

Regarding the overall mortality, we verified that it was $61.8 \%$, showing itself to be higher than that observed in two large European studies $(6,7)$ and similar to that identified in another Brazilian research (13). However, in this study, no statistically significant association was found between the timing of initiation of dialysis and death (Table 2). The criteria established for grouping patients may be one of the factors that influenced this result. The existence of several criteria for the definition of "early" and "late" promotes great methodological heterogeneity among the studied that evaluate the impact of timing of initiation of dialysis on the different outcomes of interest, hindering the in-depth analytical comparison among publications (8).

In the multicenter randomized clinical trial (RCT) Artificial Kidney Initiation in Kidney Injury, Gaudry et al (6) defined as ED that occurred within six hours after diagnosis of stage three AKI by the KDIGO criteria, whereas the diagnosis of LD considered the urinary volume, serum level of urea and potassium, $\mathrm{pH}$ or occurrence of pulmonary edema. The sample consisted of 619 patients, hospitalized in 31 ICUs in different countries. The death rate of patients in the ED group was $48.5 \%$, slightly lower than that presented by patients in the LD group, $49.7 \%(P=0.79)$. It was also verified, that the rate of catheter-related bloodstream infection was significantly higher in patients in the ED group (10\% versus 5\%, $P=$ 0.03 ). In the present study, the rate of general infections was $64 \%(n=49)$, being higher in patients in the LD group compared to the ED group ( $52 \%$ versus $71 \%, P=0.088$ ).

In another RCT, called effect of early vs delayed initiation of renal Replacement therapy on mortality in critically ill patients with AKI, Zarbock et al (7) evaluated 231 patients from a single center, defining early and LD based on time, in hours, after the AKI diagnosis in stage two and three using the KDIGO criteria. Survival after 90 days was the primary outcome analyzed, and it was found that patients in the LD group had a higher death 
Table 3. Mortality risk factors by multivariate logistic regression

\begin{tabular}{|c|c|c|c|c|c|c|}
\hline \multirow{2}{*}{ Variable } & \multicolumn{3}{|c|}{ Unadjusted model } & \multicolumn{3}{|c|}{ Adjusted model } \\
\hline & OR & $\mathrm{Cl}(95 \%)$ & $\boldsymbol{P}$ & OR & $\mathrm{Cl}(95 \%)$ & $\boldsymbol{P}$ \\
\hline Diabetes mellitus & 0.286 & $0.095-0.859$ & 0.026 & 0.496 & $0.125-1.963$ & 0.318 \\
\hline Serum creatinine $(\mathrm{mg} / \mathrm{dL})$ at ICU admission & 0.614 & $0.412-0.915$ & 0.016 & 0.453 & $0.257-0.801$ & 0.006 \\
\hline Infection & 0.237 & $0.077-0.726$ & 0.012 & 0.614 & $0.125-3.010$ & 0.547 \\
\hline \multicolumn{7}{|l|}{ Data from dialysis } \\
\hline Number of sessions & 0.949 & $0.889-1.014$ & 0.124 & - & - & - \\
\hline Total time (hours) & 0.987 & $0.969-1.005$ & 0.152 & - & - & - \\
\hline Need of interruption & 2.026 & $0.787-5.213$ & 0.143 & - & - & - \\
\hline Late dialysis & 0.413 & $0.154-1.201$ & 0.107 & - & - & - \\
\hline Length ICU stay & 0.971 & $0.947-0.996$ & 0.023 & 0.972 & $0.937-1.008$ & $0.12 \varepsilon$ \\
\hline
\end{tabular}

OR: odds ratio; $\mathrm{Cl}$ : confidence interval; ICU: intensive care unit.

rate $(54.7 \%$ versus $39.3 \%, P=0.03)$. In a second step, it was found that patients in the ED group had a higher rate of recovery of renal function after 90 days (53.6\% versus $38.7 \%, P=0.02$ ).

In a retrospective analysis of data from patients with severe AKI in another Brazilian teaching hospital, Nascimento et al (13), defining the timing of initiation of dialysis according to the blood urea nitrogen (BUN), found that patients undergoing $\mathrm{ED}(\mathrm{BUN} \leq 75 \mathrm{mg} / \mathrm{dL})$ had a lower mortality rate and a higher rate of recovery of renal function, when the timing of initiation of dialysis was determined by blood urea nitrogen levels. In the research, the multivariate analysis showed that patients in the LD group had almost four times greater chance of death than patients in the ED group $(P=0.02)$.

Unlike the cited findings, we verified that ED group presented high mortality rate compared to the LD group (Table 2). Promoting a systematic review and metaanalysis of individual data from 1879 patients with severe AKI, Gaudry et al (14) showed that the timing of initiation of dialysis does not negatively impact patient survival, and the onset can be delayed when there are no well-defined criteria for urgency in carrying out the therapy. Barbar et al (3) points out that besides not reducing the mortality of patients, ED is a treatment with potential hemorrhagic and others complication, as well as with high economic expenses which can be avoided.

Despite the recent evidence available, in clinical practice the discussion remains about the appropriate moment for starting dialysis and its effects on the mortality of patients with severe AKI in the ICU. However, other factors are involved and can also have a significant impact. In this study, the serum creatinine level, presented by patients at the time of ICU admission, was shown to be a risk factor independently associated with death (Table 3), and we verified that nonsurvivors had lower serum creatinine levels (Table 2). In another Brazilian study, this biomarker was also inversely proportional to mortality in critically ill patients with AKI (15).

Such an inverse association corroborates the possibility of late AKI diagnosis in the sample. In addition, patient malnutrition and with fluid overload is other possible justifications (15). Thinking of minimizing the impact of fluid overload on the incidence of AKI and, consequently, delaying diagnosis and worse outcomes, Macedo et al (16) and more recently Thongprayoon et al (17) proposed specific mathematical formulas for calculating creatinine in these patients. Furthermore, the influence of severe AKI on macronutrient metabolism favors hyper-catabolism, contributing to the fact that serum creatinine levels remain low (18).

Finally, we point out the theme addressed as one of the strengths of our study, especially considering that the timing when dialysis starts is a major dilemma in daily clinical practice, and its study is essential to improve support for clinical decisions taken at bedside. In addition, our study contributes to the scientific literature, because, despite the importance of the topic, its research among Brazilian working groups is still restricted and, with this, the Brazilian medical literature is considerably limited.

\section{Conclusion}

The overall and in the ED group mortality rate was elevated, however, the timing of initiation of dialysis did not show statistically significant association with death. The serum creatinine at ICU admission seems to be an important mortality predictor. In despite this, more studies are needed to better understand the impact of the timing when dialysis starts on the different outcomes of patients.

\section{Limitations of the study}

Despite of the importance of the theme and its main results, the findings of this study should not be extrapolated, given the limitations presented, among which the fact that the analyzes are based on a retrospective methodological design, with a small sample of patients admitted to a single center. Another limiting aspect refers to the criteria applied to define ED and LD. For these reasons, we encourage other primary studies to be conducted in Brazil, with more robust samples and methodological design with better levels of evidence. Thus, we will have 
a better understanding of the real impact that the timing of initiation of dialysis has on the different outcomes evaluated in patients with AKI in Brazilian ICUs.

\section{Authors' contribution}

RPS was the principal investigator of the study. LGV, DFMO, RFS and VFB were included in preparing the concept and design. ARSC and LABP revised the manuscript and critically evaluated the intellectual contents. All authors have read and approved the content of the manuscript and confirmed the accuracy or integrity of any part of the work.

Conflicts of interests

The authors declare that they have no competing interests.

Ethical considerations

Ethical issues (including plagiarism, data fabrication, double publication) have been completely observed by the authors.

\section{Funding/Support}

The study received no financial support.

\section{References}

1. Fayad All, Buamscha DG, Ciapponi A. Timing of renal replacement therapy initiation for acute kidney injury. Cochrane Cochrane Database Syst Rev. 2018;12:CD010612. doi: 10.1002/14651858.CD010612.pub2.

2. Gaudry, S, Quenot JP, Hertig A, Barbar SD, Hajage D, Ricard $J \mathrm{D}$, et al. Timing of renal replacement therapy for severe acute kidney injury in critically ill patients. Am J Respir Crit Care Med. 2019;199:1066-75. doi: 10.1164/rccm.201810-1906CP.

3. Barbar SD, Gaudry S, Dreyfuss D, Quenot JP. Renal replacement therapy: Time to give up on early initiation? Yes. Anaesth Crit Care Pain Med. 2018;37:501-3. doi: 10.1016/j. accpm.2018.11.008.

4. Meersch M, Zarbock A. Renal replacement therapy in critically ill patients: who, when, why, and how. Curr Opin Anaesthesiol. 2018;31:151-7. doi: 10.1097/ ACO.0000000000000564.

5. Romagnoli S, Clark WR, Ricci Z, Ronco C. Renal replacement therapy for AKI: When? How much? When to Stop?. Best Pract Res Clin Anaesthesiol. 2017;31:371-85. doi: 10.1016/j. bpa.2017.10.001.

6. Gaudry S, Hajage D, Schortgen F, Martin-Lefevre L, Pons B, Boulet $E$, et al. Initiation strategies for renal-replacement therapy in the intensive care unit. N Engl J Med 2016; 375:12233. doi: 10.1056/NEJMoa1603017.

7. Zarbock A, Kellum JA, Schmidt C, Aken HV, Wempe C, Pavenstädt $\mathrm{H}$, et al. Effect of early vs delayed initiation of renal replacement therapy on mortality in critically ill patients with acute kidney injury: The ELAIN randomized clinical trial.
JAMA. 2016;315:2190-9. doi: 10.1001/jama.2016.5828.

8. Moreira FT, Palomba H, Chaves RCC, Bouman C, Schultz MJ, Neto AR. Early versus delayed initiation of renal replacement therapy for acute kidney injury: an updated systematic review, meta-analysis, meta-regression and trial sequential analysis of randomized controlled trials. Rev Bras Ter Intensiva. 2018;30:376-84. doi: 10.5935/0103507X.20180054.

9. Von Elm E, Altman DG, Egger M, Pocock SJ, Gotzsche PC, Vandenbroucke JP, STROBE Initiative. The Strengthening the Reporting of Observational Studies in Epidemiology (STROBE) statement: guidelines for reporting observational studies. J Clin Epidemiol. 2008;61:344-9. doi: 10.1016/j. jclinepi.2007.11.008.

10. Kidney Disease: Improving Global Outcomes (KDIGO) Acute Kidney Injury Work Group (2012) KDIGO clinical practice guideline for acute kidney injury. Kidney Int. 2012;2:1-138.

11. Knaus WA, Draper EA, Wagner DP, Zimmerman JE. APACHE II: a severity of disease classification system. Crit Care Med. 1985;13:818-29.

12. Vincent JL, Moreno R, Takala J, Willatts S, De Mendonça A, Bruining $\mathrm{H}$, et al. The SOFA (Sepsis-related Organ Failure Assessment) score to describe organ dysfunction/failure. On behalf of the Working Group on Sepsis-Related Problems of the European Society of Intensive Care Medicine. Intensive Care Med. 1996;22:707-10. doi: 10.1007/BF01709751.

13. Nascimento GVR, Balbi AL, Ponce D, Abrão JMG. Early initiation of dialysis: mortality and renal function recovery in acute kidney injury patients. J Bras Nefrol. Oct-Dec 2012;34:337-42. doi: 10.5935/0101-2800.20120022.

14. Gaudry S, Hajage D, Benichou N, Chaibi K, Barbar S, Zarbock $A$, et al. Delayed versus early initiation of renal replacement therapy for severe acute kidney injury: a systematic review and individual patient data meta-analysis of randomised clinical trials. Lancet. 2020;395:1506-15. doi: 10.1016/S01406736(20)30531-6.

15. Souza SP, Matos RS, Barros LL, Rocha PN. Inverse association between serum creatinine and mortality in acute kidney injury. J Bras Nefrol. 2014;36:469-75. doi: 10.5935/01012800.20140067.

16. Macedo E, Bouchard J, Soroko SH, Chertow GM, Himmelfarb J, Ikizler TA, et al. Fluid accumulation, recognition and staging of acute kidney injury in critically-ill patients. Crit Care. 2010; 14:R82. doi: 10.1186/cc9004.

17. Thongprayoon C, Cheungpasitporn W, Srivali N, Ungprasert P, Kittanamongkolchai W, Kashani K. The impact of fluid balance on diagnosis, staging and prediction of mortality in critically ill patients with acute kidney injury. J Nephrol. 2016;29(2):2217. doi: 10.1007/s40620-015-0211-3.

18. Berbel MN, Pinto MPR, Ponce D, Balbi AL. Nutritional aspects in acute kidney injury. Rev Assoc Med Bras (1992). 2011;57:6006. doi: 10.1590/s0104-42302011000500022. 